\title{
Biomarkers of Cognitive Training Effects in Aging
}

\author{
Sylvie Belleville $\cdot$ Louis Bherer
}

Published online: 19 April 2012

(C) The Author(s) 2012. This article is published with open access at Springerlink.com

\begin{abstract}
An increasing number of studies have relied on brain imaging to assess the effects of cognitive training in healthy aging populations and in persons with early Alzheimer's disease or mild cognitive impairment (MCI). At the structural level, cognitive training in healthy aging individuals has been associated with increased brain volume, cortical thickness, and density and coherence of white matter tracts. At the functional level, task-related brain activation (using fMRI and PET) and fluorodeoxyglucose positron emission tomography (FDG-PET) were found to be sensitive to the effects of training. In persons with MCI, cognitive training increased brain metabolism and task-related brain activation, whereas healthy older adults showed patterns of increased and decreased activation. Further studies are required to generalize these findings to larger groups and to investigate more diverse training protocols. Research will also need to address important methodological issues regarding the use of biomarkers in cognitive aging, including reliability, clinical validity, and relevance to the pathophysiological process.
\end{abstract}

Keywords Biomarkers $\cdot$ Cognitive training $\cdot$ Cognitive intervention $\cdot$ Brain imaging $\cdot$ Aging $\cdot$ Mild cognitive

S. Belleville $(\bowtie) \cdot$ L. Bherer

Centre de Recherche,

Institut Universitaire de Gériatrie de Montréal,

4565 Chemin Queen Mary,

Montréal, Quebec H3W-1 W5, Canada

e-mail: sylvie.belleville@umontreal.ca

S. Belleville

Département de Psychologie, Université de Montréal,

Montréal, Quebec, Canada

L. Bherer

Département de Psychologie, Université du Québec à Montréal,

Montréal, Quebec, Canada impairment · Alzheimer's disease · fMRI ·

Structural imaging

\section{Introduction}

Cognitive training is designed to restore, increase, or optimize capacities in persons suffering from cognitive impairment $[1,2 \bullet \bullet$. Many studies have used strategy memory training to enhance episodic memory in older adults. Multimodal training, which combines cognitive training with psychosocial or other activities, has also gained popularity because it is often viewed as more ecological and potentially more clinically relevant. Alternatively, a great deal of studies have used specific training protocols to assess in more detail the cognitive processes that show residual plasticity as the brain matures. Many of these have reported positive effects of cognitive training in healthy older adults [3, 4] and in persons with mild cognitive impairment (MCI) [2••, $5,6 \bullet \bullet, 7,8]$, a transitional stage between normal aging and dementia. More recently, an increasing number of studies have relied on biological measures, mostly brain imaging, to assess the effect of cognitive training in aging. An analysis of the implications and implicit assumptions underlying this approach is therefore timely, particularly with the increasing importance and appeal for the identification of biomarkers.

A biomarker is a measurement used as an indicator of normal biological or disease processes. Biomarkers should meet the criteria of being a biologically plausible measure of the disease or condition and sharing a coherent relation with the disease progression and severity. For example, brain imaging allows for the measurement of structural and functional changes that are characteristic of Alzheimer's disease (AD) and that correlate with disease progression and severity. Brain imaging measures are also modified by the normal 
aging process and are related to cognitive changes that occur with age. A biomarker also can be used as "surrogate marker." Surrogate markers are used in therapeutic trials as clinically meaningful measures of the effect of a therapy $[9,10]$. Surrogate biomarkers are used when optimal clinical outcomes (for example, progression to dementia, or death) might be undesired, require too long a follow-up, or necessitate too large a sample. By using biomarkers, one can design a study where therapeutic effects are measured in a more feasible and timely manner. In the context of cognitive training, biomarkers also can provide invaluable information on the mechanisms through which an intervention enhances cognition. In addition, biomarkers can be used to quantify cognitive and neural plasticity by revealing cognitive and neural compensatory mechanisms, or by indicating patterns of changes that suggest increased efficiency of information processing in aging.

Not all biological measures are appropriate biomarkers and similarly, not all biomarkers are appropriate surrogates of training efficacy. Biomarkers need to be sensitive to the disease and to correlate with progression and severity, and their biological relation with the disease must be at least partially understood. Surrogate markers are typically biomarkers of the disease, but they also need to be sensitive to change and reliable over time, and their mechanisms should be relevant to the therapeutic effect that they are expected to indicate. In this review, we will examine structural and functional brain imaging as potential surrogate markers for cognitive training effects. We will discuss studies that have used brain imaging as a marker of cognitive training effects in healthy aging and in MCI, the early stage of $\mathrm{AD}$. We also will address some challenges, limitations, and implications of using brain imaging as biomarkers of cognitive training effects in aging populations.

\section{Structural Imaging as a Biomarker of Cognitive Training}

Structural brain imaging provides quantitative information on brain structure, including whole-brain volume, regional grey matter volumes, cortical thickness, and indicators of white matter integrity and microstructure. Brain imaging techniques have provided valuable information regarding the effects of aging on brain structure. Such studies have found that brain volume decreases with age and that those structural changes accelerate, with an annual decline of $0.35 \%$ in older adults, compared to $0.12 \%$ in young adults (see Dennis et al. [11] and Raz [12] for reviews). Normal aging does not alter all cognitive functions at the same time in the course of aging, and likewise, some brain regions are more sensitive to age than others. The caudate, cerebellum, hippocampi, and association cortices show the largest volume loss. In addition, aging brings changes in white matter integrity, with greater changes occurring after the seventh decade, and those are localized preferentially in the anterior regions (frontal and prefrontal).

Training-related structural changes often have been reported in younger adults and middle-aged participants, but very few studies have been published on the structural modifications produced by cognitive training in older adults. In one of these studies, Boyke et al. [13] showed with voxel-based morphometry (VBM) that grey matter volume increased in older adults after 3 months of learning classic three-ball cascade juggling. Changes were observed in the middle temporal area (V5) of the visual cortex, in the left hippocampus, and in the nucleus accumbens bilaterally. Interestingly, the training-related changes found in the hippocampus and nucleus accumbens were not observed in young adults. This study suggests that training can induce considerable structural plasticity in older adults, although older adults showed slightly smaller changes than younger adults. It is of note, however, that the authors did not find any correlation between changes in grey matter and performance or exercise intensity.

In a more recent study, Engvig and colleagues [14••] examined the effects of memory training on cortical thickness in middle-aged and elderly healthy volunteers. After 8 weeks of memory training using the method of loci, participants in the training group showed improved source memory and regional increases in cortical thickness of the lateral orbitofrontal cortex bilaterally and right fusiform cortex. Changes in cortical thickness in the right fusiform and lateral orbitofrontal cortex correlated positively with improvement in source memory, indicating that those structural changes are relevant markers of such cognitive changes. The training also had an impact on the alterations found in the white matter microstructure [15••]. Using diffusion tensor imaging (DTI), the authors found reduced fractional anisotropy (FA) in the frontal areas of untrained older adults, whereas no change was found in those who received memory training. Training-associated effects on FA seemed to be accompanied by a relative decrease in radial diffusivity, which might indicate a role for myelination in white matter plasticity. In line with other DTI studies showing that changes in myelination lead to FA changes in DTI,

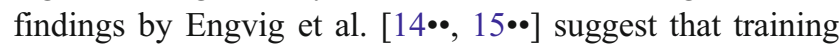
protects against age-related reductions in myelination. In this study, memory improvements were correlated with changes in anterior FA, providing support for DTI as a valid surrogate marker for the effects of cognitive training. Lovden et al. [16] also found increased FA (and decreased mean diffusivity) in older adults after a multidimensional program with repeated practice on working memory, episodic memory, and perceptual speed tasks. These changes were found in the 
anterior portion of the corpus callosum but were not correlated with cognitive improvement.

Surprisingly, no study has reported changes in structural volume or connectivity after executive control or attentional training, despite the fact that these types of process-based training have been widely used with older adults. However, an interesting set of studies suggests that structural brain imaging could be used as a biomarker of the impact of attention training. Erickson et al. [17] explored whether the effects of training with the Space Fortress video game could be predicted by the pretraining volume of either of the two key brain regions implicated in learning and memory: the striatum and the hippocampus. They observed that only dorsal striatal volumes, but not ventral striatum, predicted early acquisition rates.

Taken together, these findings suggest that structural volume in certain regions of the brain could help predict effects of cognitive training, and that cognitive training can induce short-term structural changes in older adults. Measures of regional grey matter volume with VBM, cortical thickness and white matter integrity with DTI and FA have been shown to be sensitive to cognitive training in healthy older adults. Though we found no study that had used structural brain imaging as a biomarker of training efficacy in MCI, structural brain changes have been documented in the early course of $\mathrm{AD}$. In particular, regional cortical thickness was found to be reduced in $\mathrm{MCI}$ and predicted progression from MCI to $\mathrm{AD}$ $[18,19]$. This measure might thus represent a very powerful surrogate marker to assess cognitive training effects in MCI populations as well.

\section{Functional Imaging as a Biomarker of Cognitive Training}

Functional brain imaging displays patterns of brain activation as patients perform cognitive tasks. Functional imaging has proven to be a critical tool for understanding the changes in neural mechanisms occurring in aging and $\mathrm{AD}$. For this reason, it has potential as a biomarker. It also has many of the features necessary to qualify as a valid surrogate marker in healthy aging and in early AD. First, functional brain imaging is age-sensitive. When brain imaging is used in older adults to examine changes in cerebral blood flow associated with memory tasks, it reveals a combination of increased activation patterns (eg, prefrontal activation during working memory tasks) and decreased activation patterns (eg, in the left prefrontal and median temporal lobes) or compensatory activation patterns. Compensatory patterns of activation refers to the process by which alternative or atypical brain regions compensate for reduced processing efficiency that occurs with age.
Compensatory recruitment can involve the hemispheric homologues (see HAROLD model [hemispheric asymmetry reduction in older adults] proposed by Cabeza [20]) or anterior recruitment (see PASA [posterior-anterior shift in aging] in Davis et al. [21]). Functional brain imaging also shows impairments early in the development of AD [22-24]. Reduced activation is found in the medial temporal areas but also in some regions of the prefrontal cortex of AD patients. Studies of MCI, the early stage of $\mathrm{AD}$, have reported the presence of hyperactivation early in the process, followed by hypoactivation as patients progress from MCI to AD [25-28]. Several studies [25, 29-31] have reported that brain activation differs markedly as a function of the severity of MCI, with greater brain activation found in patients with early phase MCI than at a later stage in the disease. It was also found that hippocampal activation was inversely associated with disease severity [29, 31]. O'Brien et al. [32] showed a marked decrease in activation over a 2-year period during the MCI phase. Thus, whereas AD is mostly characterized by reduced brain activation, a number of studies on MCI have reported increased activation; that is, greater task-related brain recruitment in persons with MCI than in healthy older adults. There is also accumulating evidence that activation is inversely related to the severity of symptoms and disease. Overall, milder symptoms and earlier phases of the disease are characterized by greater task-associated brain recruitment.

Functional brain imaging can thus qualify as a biomarker of aging and $\mathrm{AD}$ because it is sensitive to chronological age. It also reveals a characteristic pattern of impairment in those diagnosed with $\mathrm{AD}$ that changes with disease severity and progression. A few studies have used functional brain imaging as a biological surrogate of training efficacy in healthy aging populations and persons with MCI. In healthy aging, most studies reporting training-related changes in brain activation patterns have used memory training. Among the first studies to do so, Nyberg et al. [33] noted improved memory performance in both older and younger adults after training with a visual-based mnemonic (the method of loci). They observed increased brain activity (using positron emission tomography [PET]) during memory encoding in occipitoparietal and frontal brain regions after training in younger adults. In older adults, increased occipitoparietal activity was observed only in participants that improved after training, and no change was observed in frontal regions. Valenzuela et al. [34] used localized proton magnetic resonance spectroscopy (MRS) to measure changes in the biochemistry of the right hippocampus, midline parietaloccipital region, and left frontal lobes after memory training with the method of loci. They observed improved performance after 5 weeks of training accompanied by increased creatine and choline signals in the hippocampus. This study 
shows that focused memory exercises can induce measurable and persisting biochemical changes in the hippocampus, and that use of the memory program may have led to increased resting oxidative phosphorylation in a region that plays a critical role in memory processing.

Training-induced changes in brain activity were also reported after attention control training using computerbased programs. Erickson et al. [35] conducted a randomized longitudinal dual-task training study of functional MRI (fMRI) activation measures in older adults. They observed training-induced changes in activation in two cortical areas commonly associated with age-related atrophy: the dorsal and ventral prefrontal cortex. Interestingly, some brain regions showed equal changes among older and younger adults, whereas others showed differential training effects. For instance, in older adults, increased activity was found in the left ventrolateral prefrontal cortex (VLPFC) region (near Broca's area), suggesting an increased reliance on verbal or inner speech strategies during dual-task performance. Increased verbalization also has been reported as an efficient strategy to enhance performances in training with a switching task [36]. The authors also observed decreased activity in the right VLPFC in both younger and older adults, suggesting a reduced dependence on response selection strategies or a more efficient response-stimulus association. That both age groups showed similar reductions in activity in this region and that changes in brain activation correlated with changes in behavior suggest that patterns of changes in brain activation were important correlates of training-related improvements in behavior and age is not a factor. Brehmer et al. [37•] examined the behavioral performance and neural activity following 5 weeks of intensive working memory adaptive individualized training. Brain activity was measured before and after training, using fMRI, while participants performed a working memory task in two difficulty conditions. Neocortical brain activity decreased post-training only in the training group, which indicates intervention-related increases in neural efficiency.

A few studies have used functional brain imaging as a biological surrogate of training efficacy in MCI, and those studies indicate that fMRI is sensitive to changes following cognitive training in this group of individuals. Belleville et al. [38••] found that memory-related activation increased after strategy memory training in persons with MCI. Researchers also found that training increased activation in specialized brain regions involved in memory and in compensatory brain regions not typically activated by the verbal memory task (eg, the right inferior parietal lobe). Furthermore, relative to healthy older adults, a number of brain regions that were dysfunctional before training were no longer different following training. Importantly, activation of the right inferior parietal lobe was found to correlate with the efficacy of memory training in persons with MCI. This indicates that the biological change has a valid relation to the expected clinical outcome.
Training in this study did not increase activation of the hippocampus, which is surprising considering the importance of this structure in $\mathrm{AD}$. This might be due to the fact that the training program relied heavily on the teaching of visualbased strategies and thus promoted the recruitment of prefrontal and posterior brain regions. Hampstead et al. [39•] used a memory training method that relied on the identification of salient visual cues to learn new face-name associations. In a pilot study, they reported increased activation in the defaultmode network, which comprised the medial frontal, parietal, and occipital brain areas, as well as increased connectivity between the temporal lobe, occipital regions, and precuneus following training in MCI populations. In a subsequent study, the researchers used a form of memory training relying on mental imagery to learn object-location associations and compared it to an exposure-control condition [40••]. Before training, they found reduced hippocampal activation in MCI participants relative to controls. Hippocampal activation increased significantly following training compared to the exposure-control condition. Overall the studies of Hampstead et al. $[39 \bullet, 40 \bullet$ found changes almost exclusively in areas that showed impaired activation before the training. Rosen and collaborators [41] randomly assigned persons with MCI to either the POSIT science program (Posit Science Corporation, San Francisco, CA), designed to improve speed and temporal auditory processing, or a control condition where patients participated in diverse computerized activities. The authors reported increased left hippocampal activation in trained participants only. There was a tendency for a significant correlation between brain changes and behavioral changes, but this was not significant, perhaps due to the very small sample size.

To our knowledge, only two studies have examined brain-related changes following executive training in MCI participants. Carlson et al. [42] randomly assigned 17 individuals with low education, low income, and low minimental state examination to participate in Experience Corps, a program promoting social engagement, or to a control condition. Brain activation associated with performing an executive function test (flanker-task) had increased in the left dorsolateral prefrontal cortex and anterior cingulate gyrus in trained participants. Clare et al. [43] reported a single case study where goal-oriented training of one MCI patient led to decreased activation in sensory regions and increased activation in memory-related regions during an associative face-name association task.

PET can measure neural activity by recording the uptake of [18F]fluorodeoxyglucose (FDG). Reduced FDG-PET at rest may reveal early cerebrometabolic changes in $\mathrm{AD}$ and could represent a valid biomarker of neuronal injury in early $\mathrm{AD}$ and $\mathrm{MCI}[44,45]$. A 6-month multicomponent cognitive training program was found to reduce decline in brain glucose metabolism in MCI and early AD participants [46 ${ }^{\bullet}$. The strongest attenuated decline was found in the left 
anterior temporal lobe and left anterior cingulate in persons with MCI. However, there was no correlation between cognitive improvement and changes in glucose metabolism. In turn, Small and collaborators [47] found that a multimodal intervention (cognitive stimulation, physical activity, stress reduction, healthy diet) decreased FDG-PET uptake in the left dorsolateral prefrontal cortex of non-demented older adults. This can be interpreted as supporting more efficient processing following training in older adults, whereas the data with MCI might suggest an impact on the pathological process underlying $\mathrm{AD}$.

\section{Implications for Future Research}

The present review of recent studies provides support for the use of structural and functional brain imaging as sensitive surrogate biomarkers for the effects of cognitive training (see Table 1 for summary findings). However, further studies are required to generalize these findings to larger groups and more diverse training protocols. At the structural level, reliable training effects have been reported for regional brain volume, cortical thickness, and white matter microstructure. At the functional level, task-related brain activation (using fMRI and PET) and FDG-PET at rest were found to be sensitive to training effects. In general, cognitive training led to increased brain volume and increased density and coherence of white matter tracts. Functionally, training increased brain metabolism at rest and task-related brain activation in persons with MCI; however healthy older adults showed patterns of increased and decreased activation.

Apart from being sensitive to change, there are a number of other important characteristics to guide the use of biomarkers as surrogate markers of training efficacy. One of

Table 1 Summary of findings

\begin{tabular}{ll}
\hline Biomarker used & Effect observed \\
\hline $\begin{array}{l}\text { Grey matter volume } \\
\text { (VBM) }\end{array}$ & Increased volume \\
Cortical thickness & Increased thickness \\
White matter integrity & Increased FA \\
(DTI) & Increased creatine and choline signal \\
Biochemistry (MRS) & Reduced activation in healthy aging \\
Glucose metabolism & Increased activation in MCI \\
(FDG-PET) & Increased \& decreased activation in \\
Task-related activation & healthy aging \\
& Increased activation in MCI \\
\hline
\end{tabular}

$V B M$ voxel-based morphometry; DTI diffusion tensor imaging; $F A$ fractional anisotropy; $M R S$ magnetic resonance spectroscopy; $F D G$ $P E T$ fluorodeoxyglucose positron emission tomography; $M C I$ mild cognitive impairment these is its reliability over time. Training efficacy is assessed by repeated measurement, and thus, measures ought to be consistent at the individual and/or group level. Some of the aforementioned studies have implemented a "replication" component in their design to assess changes in an independent group of nontreated participants. For example, the replication component provided by Engvig et al. [14••], though not a reliability study as such, provides support for the use of cortical thickness as a replicable measure in healthy older adults. Studies in young adults have shown that many of the DTI measures have good test-retest reliability when controlling for motion and using a sufficient number of gradient directions [48]. Formal reliability studies have shown that fMRI is reliable for use with older adults and persons with MCI $[49,50]$. Clement and Belleville [49] found very few changes in the areas and levels of activation when comparing two measures separated by a 2-month interval, a timeframe typically found in cognitive training studies. Furthermore, it was found that persons with MCI, older adults, and young adults showed comparable overlap ratios, a reproducibility index measuring the amount of voxels activated in one versus both sessions. Importantly, however, the magnitude of the overlap ratio only indicates moderate between-session agreement. This suggests that treatment effects can be more reliably measured by comparing condition contrasts (or region of interest [ROI]) across sessions rather than by comparing voxel activation (see also Putcha et al. [50]). Similarly, intraclass correlations show important intrasubject variability, indicating that while reliable at the group level, care should be taken when interpreting fMRI treatment data at the level of individuals.

One other important characteristic when selecting a "surrogate marker" is that the selected biological measure reflects a clinically relevant change. One way to determine this is by examining the correlation between changes in the biological marker and changes in the relevant clinical or cognitive outcome. In some of these studies, correlations were found between changes in the biomarker and changes

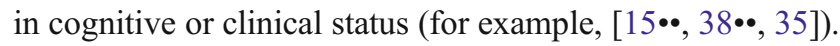
However, this was not systematically found (for example, $[13,16,41,46 \cdot])$ or even documented. Lack of a relation might be attributable to many factors, which need to be better understood, including insufficient power, inappropriate selection of clinical variables, or the presence of a nonselective effect.

Relevance also can be determined by selecting biomarkers that reflect neuropathological processes underlying the disease, and in that case, it is important to fully understand the relationship between the surrogate marker and the pathophysiology of the condition of interest [10]. Most studies have used markers that are considered valid measures of the processes that underlie normal aging or AD. However, some critical biomarkers of early AD have surprisingly not been tested as 
surrogate markers of training. One of them is beta-amyloid accumulation. It is unclear whether short-term cognitive training is likely to have an effect on beta-amyloid deposition. However, some recent studies have reported that having engaged in cognitively stimulating activities in early and middle life is associated with reduced beta-amyloid accumulation in older adults [51]. It has been suggested that the increased synaptic activity provided by cognitive stimulation might protect against amyloid deposition.

\section{Conclusions}

In conclusion, this review supports the notion that brainimaging techniques could be reliably used to assess trainingrelated changes in brain structure and function in healthy older adults and patients with MCI or AD. However, future studies are needed to identify all the potential biomarkers and surrogate biomarkers of brain plasticity induced by cognitive training interventions. We believe that following a structured methodology that meets the prerequisites provided in the present review would effectively guide future research and help to increase the body of knowledge on brain changes associated with cognitive training in older adults.

Acknowledgements The authors thank Amanda De Fillipo for editing the paper and Émilie Lepage for helping in the preparation of the manuscript.

Disclosures Dr. Sylvie Belleville has received payment from Bordeaux University related to training for the MEMO program. L. Bherer: none.

Open Access This article is distributed under the terms of the Creative Commons Attribution License which permits any use, distribution, and reproduction in any medium, provided the original author(s) and the source are credited.

\section{References}

Papers of particular interest, published recently, have been highlighted as:

- Of importance

•. Of major importance

1. Belleville $\mathrm{S}$. Cognitive training for persons with mild cognitive impairment. Int Psychogeriatr. 2008;20(1):57-66.

2. • Lustig C, Shah P, Seidler R, Reuter-Lorenz PA. Aging, training, and the brain: a review and future directions. Neuropsychol Rev. 2009;19(4):504-22. This excellent paper reviews the literature on cognitive training and discusses the implication for current models of brain aging and for brain-behavior relationships.

3. Ball K, Berch DB, Helmers KF, et al. Effects of cognitive training interventions with older adults: a randomized controlled trial. JAMA. 2002;288(18):2271-81.
4. Willis SL, Tennstedt SL, Marsiske M, et al. Long-term effects of cognitive training on everyday functional outcomes in older adults. JAMA. 2006;296(23):2805-14.

5. Belleville S, Gilbert B, Fontaine F, et al. Improvement of episodic memory in persons with mild cognitive impairment and healthy older adults: evidence from a cognitive intervention program. Dement Geriatr Cog Disord. 2006;22:486-99.

6. •• Mowszowski L, Batchelor J, Naismith SL. Early intervention for cognitive decline: can cognitive training be used as a selective prevention technique? Int Psychogeriatr. 2010;22(4):537-48. This is an excellent paper for the reader who wants to get acquainted with the literature on training as a prevention for cognitive declines in aging and in persons at risk for Alzheimer's disease.

7. Stuss DT, Robertson IH, Craik FI, et al. Cognitive rehabilitation in the elderly: a randomized trial to evaluate a new protocol. J Int Neuropsychol Soc. 2007;13(1):120-31.

8. Troyer AK, Murphy KJ, Anderson ND, et al. Changing everyday memory behaviour in amnestic mild cognitive impairment: a randomised controlled trial. Neuropsychol Rehabil. 2008;18(1):65-88.

9. Katz R. Biomarkers and surrogate markers: an FDA perspective. NeuroRx. 2004;1(2):189-95.

10. Fleming TR, DeMets DL. Surrogate end points in clinical trials: are we being misled? Ann Intern Med. 1996;125(7):605-13.

11. Dennis NA, Kim H, Cabeza R. Age-related differences in brain activity during true and false memory retrieval. J Cogn Neurosci. 2008;20(8):1390-402.

12. Raz N. The aging brain observed in vivo: differential changes and their modifiers. In: Cabeza R, Nyberg L, Park DC, editors. Cognitive neuroscience of aging. New York: Oxford University Press; 2005. p. 19-57.

13. Boyke J, Driemeyer J, Gaser C, et al. Training-induced brain structure changes in the elderly. J Neurosci. 2008;28(28):7031-5.

14. •- Engvig A, Fjell AM, Westlye LT, et al. Effects of memory training on cortical thickness in the elderly. NeuroImage. 2010;52(4):1667-76. The authors report change in cortical thickness induced by memory training. Structural changes positively correlated with improvement in source memory indicate that those structural changes are relevant markers of the expected underlying cognitive changes.

15. •• Engvig A, Fjell AM, Westlye LT et al. Memory training impacts short-term changes in aging white matter: a Longitudinal Diffusion Tensor Imaging Study. Hum Brain Mapp 2011. This is one of the few studies investigating the effect of cognitive training on the microstructure of white matter using diffusion tensor imaging.

16. Lovden M, Bodammer NC, Kuhn S, et al. Experience-dependent plasticity of white-matter microstructure extends into old age. Neuropsychologia. 2010;48(13):3878-83.

17. Erickson KI, Boot WR, Basak C, et al. Striatal volume predicts level of video game skill acquisition. Cereb Cortex. 2010;20(11):2522-30.

18. Julkunen V, Niskanen E, Koikkalainen J, et al. Differences in cortical thickness in healthy controls, subjects with mild cognitive impairment, and Alzheimer's disease patients: a longitudinal study. J Alzheimers Dis. 2010;21(4):1141-51.

19. Julkunen V, Niskanen E, Muehlboeck S, et al. Cortical thickness analysis to detect progressive mild cognitive impairment: a reference to Alzheimer's disease. Dement Geriatr Cogn Disord. 2009;28(5):404-12.

20. Cabeza R. Hemispheric asymmetry reduction in older adults: the HAROLD model. Psychol Aging. 2002;17(1):85-100.

21. Davis SW, Dennis NA, Daselaar SM, et al. Que PASA? The posterior-anterior shift in aging. Cereb Cortex. 2008;18(5):1201-9.

22. Devous Sr MD. Functional brain imaging in the dementias: role in early detection, differential diagnosis, and longitudinal studies. Eur J Nucl Med Mol Imaging. 2002;29(12):1685-96.

23. Schwindt GC, Black SE. Functional imaging studies of episodic memory in Alzheimer's disease: a quantitative meta-analysis. NeuroImage. 2009;45(1):181-90. 
24. Villain N, Chetelat G, Desgranges B, Eustache F. Neuroimaging in Alzheimer's disease: a synthesis and a contribution to the understanding of physiopathological mechanisms. Biol Aujourdhui. 2010;204(2):145-58.

25. Celone KA, Calhoun VD, Dickerson BC, et al. Alterations in memory networks in mild cognitive impairment and Alzheimer's disease: an independent component analysis. J Neurosci. 2006;26 (40):10222-31.

26. Dickerson BC, Sperling RA. Functional abnormalities of the medial temporal lobe memory system in mild cognitive impairment and Alzheimer's disease: insights from functional MRI studies. Neuropsychologia. 2008;46(6):1624-35.

27. Pihlajamaki M, Jauhiainen AM, Soininen H. Structural and functional MRI in mild cognitive impairment. Curr Alzheimer Res. 2009;6(2):179-85.

28. Sperling R. Functional MRI studies of associative encoding in normal aging, mild cognitive impairment, and Alzheimer's disease. Ann N Y Acad Sci. 2007;1097:146-55.

29. Clement F, Belleville S. Effect of disease severity on neural compensation of item and associative recognition in mild cognitive impairment. J Alzheimers Dis. 2012;29(1):109-23.

30. Clement F, Belleville S, Mellah S. Functional neuroanatomy of the encoding and retrieval processes of verbal episodic memory in MCI. Cortex. 2010;46(8):1005-15.

31. Clement F, Belleville S. Compensation and disease severity on the memory-related activations in mild cognitive impairment. Biol Psychiatry. 2010;68(10):894-902.

32. O'Brien JL, O'Keefe KM, LaViolette PS, et al. Longitudinal fMRI in elderly reveals loss of hippocampal activation with clinical decline. Neurology. 2010;74(24):1969-76.

33. Nyberg L, Sandblom J, Jones S, et al. Neural correlates of trainingrelated memory improvement in adulthood and aging. Proc Natl Acad Sci U S A. 2003;100(23):13728-33.

34. Valenzuela MJ, Jones M, Wen W, et al. Memory training alters hippocampal neurochemistry in healthy elderly. Neuroreport. 2003;14(10):1333-7.

35. Erickson KI, Colcombe SJ, Wadhwa R, et al. Training-induced plasticity in older adults: effects of training on hemispheric asymmetry. Neurobiol Aging. 2007;28(2):272-83.

36. Kray J, Lucenet J, Blaye A. Can older adults enhance taskswitching performance by verbal self-instructions? The influence of working-memory load and early learning. Front Aging Neurosci. 2010;2(147):1-9.

37. • Brehmer Y, Rieckmann A, Bellander M, et al. Neural correlates of training-related working-memory gains in old age. NeuroImage. 2011;58(4):1110-20. This study elegantly shows neocortical brain activity changes post-training, specific to the training group, which suggests intervention-related increases in neural efficiency.

38. •• Belleville S, Clement F, Mellah S et al. Training-related brain plasticity in subjects at risk of developing Alzheimer's disease. Brain 2011. This is one of the first papers to report the effect of a multifactorial memory training program on the brain activation of persons with MCI. It shows that cognitive training increases brain activation and that the increase in activation is correlated with performance.
39. - Hampstead BM, Stringer AY, Stilla RF, et al. Activation and effective connectivity changes following explicit-memory training for face-name pairs in patients with mild cognitive impairment: a pilot study. Neurorehabil Neural Repair. 2011;25(3):210-22. This is one of the first papers to report the effect of an associative memory training program on the brain activation of persons with MCI.

40. • Hampstead BM, Stringer AY, Stilla RF et al. Mnemonic strategy training partially restores hippocampal activity in patients with mild cognitive impairment. Hippocampus 2012. In this paper, the authors report that memory training can have a direct effect on hippocampal activity in persons with MCI.

41. Rosen AC, Sugiura L, Kramer JH, et al. Cognitive training changes hippocampal function in mild cognitive impairment: a pilot study. J Alzheimers Dis. 2011;26 Suppl 3:349-57.

42. Carlson MC, Erickson KI, Kramer AF, et al. Evidence for neurocognitive plasticity in at-risk older adults: the experience corps program. J Gerontol Biol Med Sci. 2009;64(12):1275-82.

43. Clare L, van Paasschen J, Evans SJ, et al. Goal-oriented cognitive rehabilitation for an individual with Mild Cognitive Impairment: behavioural and neuroimaging outcomes. Neurocase. 2009;15 (4):318-31.

44. Albert MS, DeKosky ST, Dickson D, et al. The diagnosis of mild cognitive impairment due to Alzheimer's disease: recommendations from the National Institute on Aging-Alzheimer's Association workgroups on diagnostic guidelines for Alzheimer's disease. Alzheimers Dement. 2011;7(3):270-9.

45. McKhann GM, Knopman DS, Chertkow H, et al. The diagnosis of dementia due to Alzheimer's disease: recommendations from the National Institute on Aging-Alzheimer's Association workgroups on diagnostic guidelines for Alzheimer's disease. Alzheimers Dement. 2011;7(3):263-9.

46. - Forster S, Buschert VC, Teipel SJ, et al. Effects of a 6-month cognitive intervention on brain metabolism in patients with amnestic MCI and mild Alzheimer's disease. J Alzheimers Dis. 2011;26 Suppl 3:337-48. This study reports increased brain metabolism in persons with $M C I$ and mild AD following cognitive intervention. The study is interesting because it relies on FDG-PET, a typical biomarker of $A D$.

47. Small GW, Silverman DH, Siddarth P, et al. Effects of a 14-day healthy longevity lifestyle program on cognition and brain function. Am J Geriatr Psychiatry. 2006;14(6):538-45.

48. Wang JY, Abdi H, Bakhadirov K, et al. A comprehensive reliability assessment of quantitative diffusion tensor tractography. NeuroImage. 2011;60(2):1127-38.

49. Clement F, Belleville S. Test-retest reliability of fMRI verbal episodic memory paradigms in healthy older adults and in persons with mild cognitive impairment. Hum Brain Mapp. 2009;30 (12):4033-47.

50. Putcha D, O'Keefe K, LaViolette P, et al. Reliability of functional magnetic resonance imaging associative encoding memory paradigms in non-demented elderly adults. Hum Brain Mapp. 2011;32 (12):2027-44.

51. Landau SM, Marks SM, Mormino EC et al. Association of lifetime cognitive engagement and low beta-amyloid deposition. Arch Neurol 2012. 